\title{
The field dependence/independence cognitive style does not control the spatial perception of elevation
}

\author{
TODD E. HUDSON, WENXUN LI, and LEONARD MATIN \\ Columbia University, New York, New York
}

\begin{abstract}
Earlier work described the presence of a significant connection between an individual's ability to disregard distracting aspects of a visual field in the classical rod-and-frame test (RFT), in which a subject is required to set a rod so that it will appear vertical in the presence of a square frame that is roll tilted from vertical, and in paper-and-pencil tests, in which the subject is required to find a hidden figure embedded in a more complex figure (the Embedded Figures Test [EFT]; see, e.g., Witkin, Dyk, Faterson, Goodenough, \& Karp, 1962; Witkin et al., 1954; Witkin, Oltman, Raskin, \& Karp, 1971). This has led to a belief in the existence of a bipolar dimension of cognitive style that is utilized in such disembedding tasks - namely, the extent to which an individual is dependent on or independent from the influence of a distracting visual field. The influence of an inducing visual field on the perception of elevation measured by the setting of a visual target to appear at eye level (the visually perceived eye level [VPEL] discrimination) has also been found to be correlated with the RFT. We have thus explored the possible involvement of the dependence/independence cognitive style on the VPEL discrimination. Measurements were made on each of 18 subjects ( 9 of them female, 9 male) setting a small target to the VPEL in the presence of a pitched visual field across a range of six pitches from $-30^{\circ}$ (topbackward) to $+20^{\circ}$ (topforward) and on each of three tests generally recognized as tests of cognitive spatial abilities: the EFT, the Gestalt Completion Test, and the Snowy Pictures Test (SPT). Although there were significant pairwise correlations relating performance on the three cognitive tests $(+.73,+.48$, and +.71 ), the correlation of each of these three with the slope of the VPEL-versus-pitch function was not significant, as it was with the slope of the perception of visual pitch of the field (PVP)-versus-pitch function. VPEL, PVP, and a cognitive factor separated into three essentially independent factors in a multiple-factor analysis, with the three cognitive tests clustering at the cognitive factor, and with no significant loading on either of the other two factors. From the above considerations and a multiple-factor analytic treatment including additional results from this and other laboratories, we conclude that the cognitive-processing style held to be involved in the performance on the EFT and the perception of vertical as measured by the RFT is not general for egocentric space perception; it does not involve the perception of elevation.
\end{abstract}

Although obviously very different in many ways, both the classical rod-and-frame test (RFT; Witkin \& Asch, 1948), in which an observer sets the orientation of a rod to appear vertical within the frontoparallel plane of a rolltilted visual field, and the Embedded Figures Test (EFT; Witkin, 1950), in which a subject attempts to find a simple outline figure within a complex visual field, require subjects to report information regarding an aspect of a visual field whose organization disrupts acquisition of veridical information. Consequently, the RFT and EFT have often been referred to as perceptual disembedding tasks. The widely-referred-to report of a significant correlation between performance on the two tasks by Witkin and his

The work described in this article was supported by Grant EY10534 from the National Eye Institute, NIH. Correspondence concerning this article should be addressed to T. E. Hudson, Department of Psychology, New York University, 6 Washington Place, New York, NY 10003 (e-mail: hudson@cns.nyu.edu). colleagues (Witkin et al., 1954) led them to propose a theoretical treatment in which they suggested that the correlation resulted from individual differences in cognitive style that involved differences in disembedding ability; this approach has been taken by others as well (regarding the EFT, see Bailey, Hustmyer, \& Kristofferson, 1961; Karp, 1963; Karp, Poster, \& Goodman, 1963; Rhodes, Carr, \& Jurji, 1968; Shade, 1984; Witkin, 1950; Witkin, Karp, \& Goodenough, 1959; regarding the RFT, see Bennett, 1956; Naylor, 1965; Witkin, 1949a; Witkin \& Asch, 1948; regarding both the EFT and the RFT, see Pizzamiglio \& Zoccolotti, 1981; Witkin, 1977; Witkin \& Goodenough, 1977; Witkin et al., 1954; Witkin, Oltman, Raskin, \& Karp, 1971). Initially formulated as a theory that held that the individual difference in performances on both the RFT and the EFT resulted from differences in the extent to which individuals were influenced by stimulation originating in the visual field or from their own bodies, a later development of the dependence/independence theory suggested that the preponderance of cues used by individuals with a differen- 
tiated $^{1}$ cognitive style were internal, producing a performance that was relatively independent of influence from the visual field for disembedding tasks; individuals with a relatively undifferentiated cognitive style were assumed to rely to a greater extent on environmental cues derived from the prevailing visual field in structuring their performance, leading to relatively visual-field-dependent performance on disembedding tasks.

Over the considerable span of time since the original report of the relation between the RFT and the EFT, a substantial expansion of the number and variety of fields to which this approach has been applied has taken place (see Witkin, 1977), and the terminology defining it has shifted as the work has grown in scope. Thus, the term field dependence/independence (FDI) was originally applied to the extent to which a subject's perception of the vertical was influenced by the surrounding visual field, and subjects whose perceptions, as measured either by a setting of a rod to appear vertical or by the setting of their own bodies to feel vertical, manifested large shifts toward the orientation of the tilted visual field were labeled field dependent, whereas subjects whose perceptions remained close to the true vertical were labeled field independent. Subsequently, the terms differentiation, disembedding, cognitive style, and cognitive restructuring were employed in a variety of contexts and were sometimes employed conjointly or interchangeably. This was a result of the overriding belief in the field that the various measures of behavior under discussion were manifestations of a stable trait that was sometimes viewed as a unitary cognitive characteristic or an aspect of personality. We have employed the term field dependence/independence in the title because it is most recognizable and seems to encompass all of the rest, but whenever feasible in the remainder of this article, we will employ cognitive restructuring as most appropriate to the concerns of the present experiments.

The large individual differences in the RFT provided the opportunity for correlational studies, and the hypothesis that these individual differences might be indicative of a more general aspect of cognition led Witkin (1950) to develop the EFT by employing the approach taken by Gottschaldt to demonstrate gestalt laws of perceptual organization by "hiding" a simple test figure within a more complex figure (Gottschaldt, 1926). The EFT originally employed by Witkin (1950) contained some of the original Gottschaldt figures and some that were developed by Witkin (1950); the test itself consisted of measurements of the time to discovery of the simple figure within the complex one for a set of figures, and Witkin et al. (1954) correlated performance on the EFT with performance on the RFT.

The theoretical approach that led to the attempt to connect the RFT and the EFT can also be applied to the egocentric perception of spatial dimensions other than the perception of verticality and, most particularly, of dimensions for which inducing stimuli from the visual field could be demonstrated to have large systematic effects that are variable across individuals, as they do for the perception of verticality. For such dimensions, the theoretical approach to FDI would suggest that analogous manipula- tions of the inducing field might produce effects that are mediated by the same differences among individuals in cognitive style as those that have been suggested to be significant for the perception of verticality. The perception of elevation is such a dimension; large systematic effects on the setting of a visual target to appear at eye level (visually perceived eye level [VPEL] discrimination) are generated by changes in the pitch of a visual field (Hudson, Li, \& Matin, 2000; Li, Dallal, \& Matin, 2001; Li \& Matin, 1996, 1998; Matin \& Fox, 1986, 1989; Matin \& Li, 1992a, 1992b, 1994a, 1994b, 1995, 1999, 2000, 2001; Poquin, Ohlmann, \& Barraud, 1998; Post, Teague, Welch, \& Hudson, 2003; Post \& Welch, 1996; Post, Welch, \& Clark, 2000; Stoper $\&$ Cohen, 1989, 1991); these effects are nearly linear with visual pitch over a range between $-40^{\circ}$ and $+30^{\circ}$. Both setting a target to VPEL in the presence of a pitched visual field and setting a rod to appear vertical in the presence
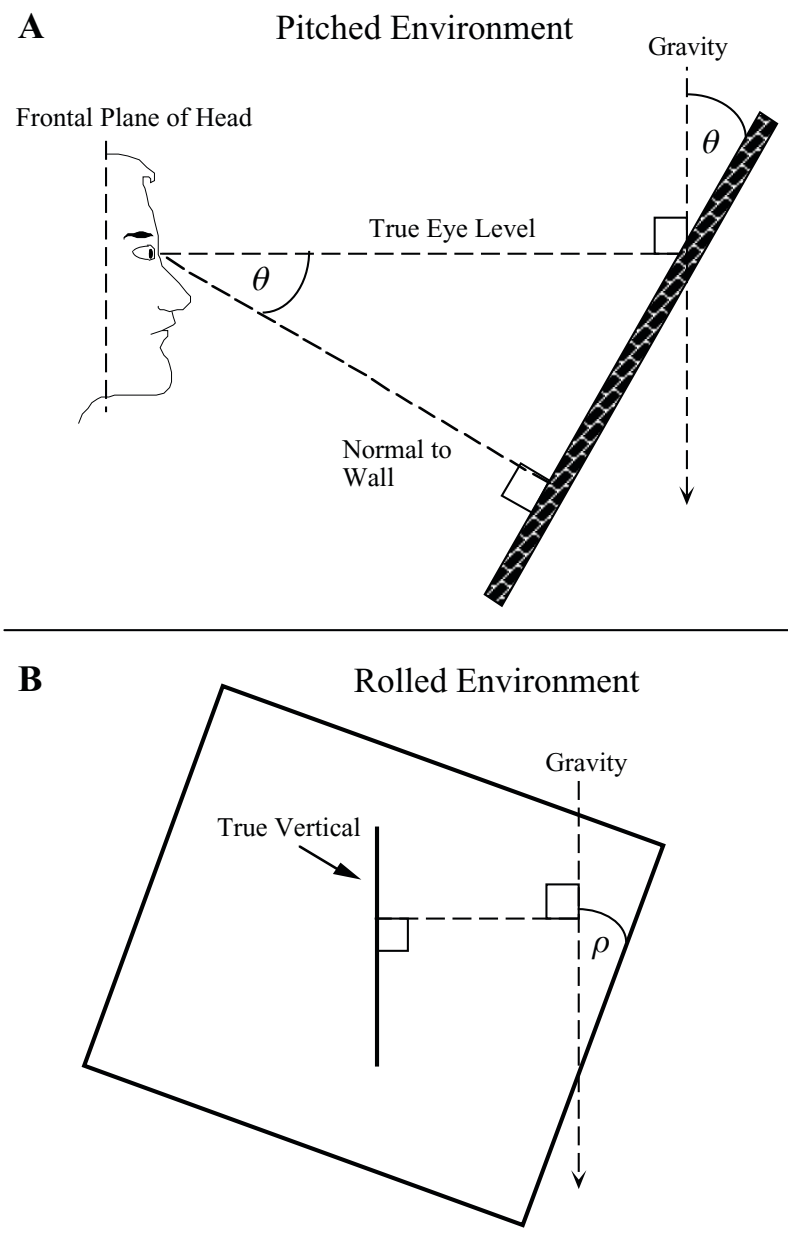

Figure 1. (A) Schematic of the relationship between the pitch $(\theta)$ of a visual field and the angular difference between the normal to the pitched plane and the observer's true eye level in a pitched environment (pitched visual field). (B) Schematic of the relationship between the roll $(\rho)$ of a visual field and the angular difference between the true vertical, defined gravitationally, and the main lines of the rolled visual field. 
of a roll-tilted visual field - the visually perceived vertical (VPV) discrimination employed in the RFT - involve significant discordances between visual and gravitational stimulation, although in different spatial dimensions (see Figure 1). In support of the possibility of a correlation between the VPEL task and the EFT is the fact that both the VPV and the VPEL discriminations are sensitive to induction by the very same variably oriented single lines from the very same planes (either erect and frontoparallel or pitched from vertical), despite differences in the planes from which the distal visual stimulation employed in the original work on VPV and VPEL arose - frontoparallel for VPV (Witkin \& Asch, 1948) and pitched from vertical for VPEL (Matin \& Li, 1994a, 1994b, 1999). In further support of a possible connection between VPV and VPEL discriminations, three different laboratories have measured significant correlations across subjects between the VPV and VPEL discriminations of roughly the same order: Poquin et al. (1998), Guzy, Cohen, and Ebenholtz (2003), and Shavit, Li, Semanek, and Matin (2004).

In combination with the previous work by Witkin and his colleagues relative to the disembedding requirement, these correlations in susceptibility to induction for the two discriminations and the considerable formal similarities between the VPV and the VPEL tasks encouraged us to search for a relation between the performances on the VPEL and EFT tasks similar to that previously reported between the VPV and the EFT tasks. The characteristics of the inducing stimulus described above, as well as the methodology and the experimental results for the VPEL discrimination, are closely analogous to those for the VPV discrimination where VPV settings are close to veridical in normally oriented environments but change with the roll tilt of the visual field as a near-linear function of the magnitude of the roll tilt deviation from an erect orientation $\left(-22^{\circ} \leq\right.$ roll $\leq+22^{\circ}$; Asch \& Witkin, 1948; Ebenholtz, 1977; Ebenholtz \& Benzschawel, 1977; Howard, 1986; Howard \& Templeton, 1966; Li \& Matin, 2005a, 2005b, 2005c; Matin \& Li, 1994b, 1995; Morant \& Beller, 1965; Wenderoth, 1982; Witkin \& Asch, 1948). It is of particular significance here that in both discriminations, subjects display large individual differences that are consistent over long periods (Matin \& Li, 1999, 2001; Witkin, 1949a). We use the gain of the VPEL-versus-pitch function (i.e., the slope of the function) to describe the magnitudes of the effects for any given subject, with relatively large gains (closer to 1) indicating subjects who would be considered field dependent and relatively small gains (closer to 0 ) indicating subjects who would be considered field independent. Field-dependent observers are, by definition, strongly influenced by the visual field: Thus, for them, the pitch of the visual field would be expected to exert a significant effect on the VPEL, driving the VPEL toward large deviations from the true eye level (TEL). Field-independent observers, on the other hand, would be less strongly influenced by the visual field and more influenced by their contributions from the body-referenced mechanism. ${ }^{2}$

In the present experiments, following the earlier work of Witkin and his colleagues, we compared the results for
VPEL with measurements on the EFT for the same subjects and also included two other tests that have been presumed to measure aspects of cognitive style very similar to those measured by the EFT: the Gestalt Completion Test (GCT; Street, 1931/1972) and the Snowy Pictures Test (SPT). The EFT is the well-known test that employs adaptations of the original Gottschaldt figures (Gottschaldt, 1926), in which a target figure shown to a subject is to be discovered in a more complex figure that has its own shape and tends to mask the presence of the target. The adaptation employed in the EFT (originated in Witkin, 1950) involves coloring various regions of the complex figures in an attempt to further obscure the location of the simple figure within it. The EFT utilizes this technique of obscuring the location of the simple figure in all but one of the test items (shown in Figure 2). The GCT is adapted from a set of figures taken from a dissertation by Street (1931/1972), in which major segments of a well-known object are deleted and the remainder displayed in normal relation to each other; the subject is required to identify the object. The SPT is similar to the GCT, but the identity of test items is obscured through both fragmentation of line drawings and the addition of small line segments ("snow") within and around the fragmented drawings. Since the GCT and the SPT provide different sorts of interference from within the visual field than does the EFT, as well as differing from each other, each of the three tasks might require somewhat different abilities for their completion. Employing all three cognitive tasks thus provided some opportunity to assess the generality of any conclusions that might be derived from an analysis in terms of disembedding based on the use of the EFT alone. From a disembedding analysis of the VPEL task given above, we would expect there to be a direct relationship between performances on the VPEL task and on each of the three cognitive tasks. Fielddependent subjects, as assessed by performance on each of the cognitive tasks, would also be expected to display field dependence, as assessed by performance on the VPEL discrimination. That is, subjects producing large VPEL/ physical pitch slopes should have relatively large average EFT completion times and small numbers of correct items on both the GCT and the SPT, whereas subjects with small VPEL/physical pitch slopes should have relatively short average EFT times and large numbers of correct items on the GCT and the SPT. We therefore tested all the subjects on all four tasks (VPEL, EFT, GCT, and SPT). The magnitudes of the correlations between VPEL slopes and scores on each of the three cognitive tasks measure the extent to which the influence of the visual field on perception was mediated by processes that were common to the VPEL discrimination in a pitched environment and the identification of simple figures in a complex visual field. As a fifth independent variable, a full set of measurements were made of the same subjects' perceptions of the pitch of the pitchroom (PVP) in which the VPEL measurements were made. These measurements serve as a second, separate measure of a different dimension of egocentric space perception and broaden the possibility of understanding the relation between space perception and cognitive re- 
Gestalt Completion Test

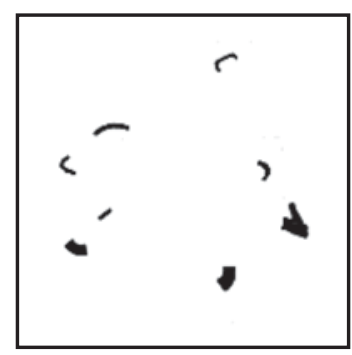

7.

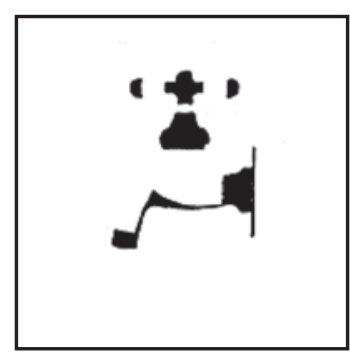

8
Snowy Pictures Test

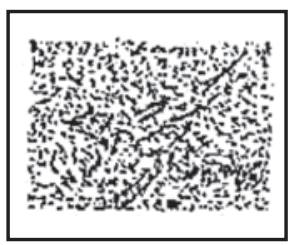

15

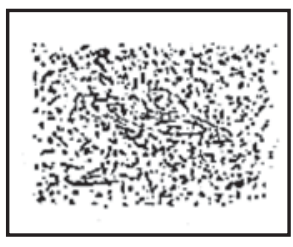

24.

\section{Embedded Figures Test}

\section{COMPLEX Figure}

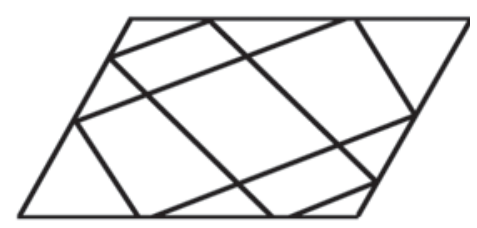

Simple Figure

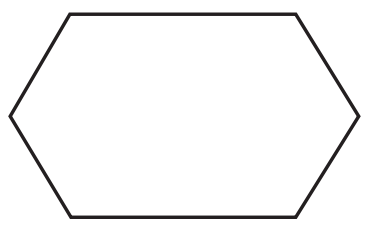

Figure 2. Examples of each of the cognitive tests. The Gestalt Completion example items are correctly identified as (7) cart and (8) faucet. The Snowy Pictures example items are correctly identified as (15) bird and (24) planet.

structuring. The relation between the PVP and the VPEL results has been fully described in a previous publication (Hudson et al., 2000). The method and results of the PVP measurements will be summarized in the Discussion section below, where those results will be included in several aspects of the theoretical treatment given there.

\section{METHOD}

\section{Subjects}

Eighteen subjects ( 9 of them male, 9 female), responding to fliers posted at the Columbia University campus, participated in the study. The subjects were paid for their participation and ranged from 18 to 33 years in age.

\section{Visually Perceived Eye Level Discrimination}

Each subject set a laser pointer to appear at the elevation corresponding to eye level under six orientations $\left(0^{\circ}, \pm 10^{\circ}, \pm 20^{\circ}\right.$, and $-30^{\circ}$ of pitch) of the illuminated pitchroom (Figure 3 ) within a single session. The order of topforward and topbackward ( + and - , respectively) orientations was randomized differently for each subject. The session included measurements with the erect orientation at three points during the session: at the beginning, middle, and end of the session. VPEL measurements were made on two successive trials at each orientation before shifting to another orientation. The randomization procedure generated a given nonzero orientation twice during the session, and so a total of four measurements were made at each nonzero pitch and six measurements in the erect pitchroom.

The VPEL discrimination was performed with the subject facing the far wall of the pitchroom (as in Figure 3), upon which the point of laser light (the target) was projected. The subjects sat with their chins supported on a chinrest mounted on a stool. They were instructed to make the VPEL discrimination by verbally indicating that the experimenter move a dim red target up or down until it appeared to lie at eye level. The target was derived from an attenuated $0.95-\mathrm{mW}$ He-Ne laser mounted behind the subject and projected onto the pitched wall within the subject's midsagittal plane. Eye level was defined for each subject by the experimenter as the point at which a line perpendicular to gravity that was projected from the eye would intersect the far wall of the pitchroom.

A psychophysical method of adjustment with hunting was employed for each trial. The experimenter asked the subjects to close their eyes while the target was being moved up or down. The subjects indicated the desired direction of movement by up or down com-

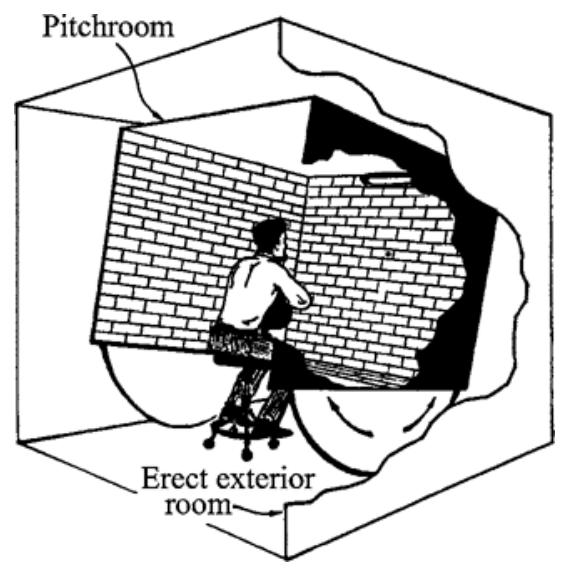

Figure 3. Artist's rendition of the pitchroom apparatus. 
mands, to which the experimenter responded by moving the laser approximately $5^{\circ}$ in the direction indicated, until smaller adjustments were required. Each time the subjects indicated that smaller adjustments were required, the experimenter would move the laser approximately half the distance of the previous adjustment. The procedure terminated when the subjects reported that the target appeared to be at eye level. For one of the two measurements, during each presentation of a given room pitch, the initial position of the target was presented above the region of uncertainty; for the other measurement, the initial position was below the region of uncertainty.

\section{Cognitive Tasks}

The three tests of cognitive ability employed in the present experiments were versions purchased from the Educational Testing Service at Princeton and are described in Ekstrom, French, Harman, and Derman (1976). The standard form of the EFT (Witkin et al., 1971) was used (see Figure 2 for an example of an EFT item). No modifications to the standard EFT instructions or scoring were made. The GCT was an adaptation from a dissertation (Street, 1931/1972). The SPT was developed by John French (A. R. Darlington, Educational Testing Service, personal communication, October 2002).

Embedded Figures Test. Each subject's EFT score was the arithmetic average of the time (in seconds) it took to find each of 12 geometric objects (simple figures) within a more complex geometric object (complex figures; see Figure 3). Each of the 12 items was presented individually. Complex and simple figures were never visible to the subjects at the same time, although the subjects were able to look back at the simple figure (while the complex figure was covered) at any time they chose. While the subjects were taking another look at the simple figure, the clock was stopped. If the subjects had not found the simple figure within $3 \mathrm{~min}$, they were stopped and were asked to continue with the next item and were given the maximum score of $180 \mathrm{sec}$ for the skipped item.

Gestalt Completion Test (Ekstrom et al., 1995). The subjects were given a set of 20 picture fragments (contained on four sheets of paper, laid out on a desk so that no sheet was covered by another; see Figure 3 for an example item) and were required to write the names of the objects depicted below the pictures. Synonyms (e.g., chair/stool, or bunny/rabbit) were accepted. The subjects were given $2 \mathrm{~min}$ in which to name the objects, in any order they chose, and were stopped at the end of the $2 \mathrm{~min}$. Their score on the GCT was the number of correctly identified items. The subjects were not required to produce a guess for items whose identities were uncertain, although this was not discouraged.

Snowy Pictures Test (Ekstrom et al., 1995). The pictures in the SPT were very similar to those in the GCT, except that there were extra short unconnected lines added to the drawings, creating noise in the form of "snow" around and over the pictures themselves (see Figure 3 for an example item). The procedure was the same as that for the GCT, except that there were 24 objects contained on two sheets of paper and the subjects were given 3 min to complete the set. The subjects were not required to produce a guess for items whose identities were uncertain, although this was not discouraged.

\section{RESULTS}

\section{Embedded Figures Test}

The average time required for the subjects to complete all 12 items was $392.1 \mathrm{sec}$, or an average of $32.7 \mathrm{sec}$ per item. The total completion times for the EFT ranged from 162 to $1,171 \mathrm{sec}$, with a median score of $255.5 \mathrm{sec}$. Of the 18 subjects, 7 ran out of time at least once (by the 3 -min rule) and proceeded to the next item without finding the simple figure within the complex figure. Of these subjects, only 2 had this happen more than once ( 1 had
Table 1

The Visually Perceived Eye Level (VPEL), Gestalt Completion Test (GCT), Snowy Pictures Test (SPT), and Embedded Figures Test (EFT) Scores for Each Subject

\begin{tabular}{|c|c|c|c|c|}
\hline Subject & $\begin{array}{l}\text { VPEL/Pitch } \\
\text { Slope ( }{ }^{\circ} \text { Visual } \\
\text { Angle/ }{ }^{\circ} \text { Pitch) }\end{array}$ & $\begin{array}{c}\text { GCT } \\
\text { (Items } \\
\text { Correct) }\end{array}$ & $\begin{array}{c}\text { SPT } \\
\text { (Items } \\
\text { Correct) }\end{array}$ & $\begin{array}{r}\text { EFT } \\
(\mathrm{Sec})\end{array}$ \\
\hline 1. J.H. & .58 & 12 & 14 & 44.6 \\
\hline 2. R.D. & .72 & 14 & 14 & 14.1 \\
\hline 3. T.F. & .71 & 11 & 16 & 22.6 \\
\hline 4. S.T. & .66 & 11 & 9 & 13.8 \\
\hline 5. M.R. & .60 & 7 & 7 & 97.6 \\
\hline 6. L.L. & .78 & 6 & 5 & 44.7 \\
\hline 7. J.P. & .63 & 11 & 16 & 18.1 \\
\hline 8. M.G. & .77 & 10 & 10 & 71.7 \\
\hline 9. E.A. & .50 & 13 & 12 & 17.7 \\
\hline 10. M.S. & .64 & 12 & 15 & 38.1 \\
\hline 11. L.S. & .80 & 14 & 17 & 15.3 \\
\hline 12. K.N. & .33 & 13 & 11 & 14.8 \\
\hline 13. J.S. & .56 & 15 & 19 & 20.0 \\
\hline 14. L.P. & .61 & 13 & 15 & 29.3 \\
\hline 15. C.T. & .76 & 15 & 13 & 13.5 \\
\hline 16. B.B. & .74 & 9 & 14 & 60.7 \\
\hline 17. N.I. & .53 & 11 & 13 & 32.5 \\
\hline 18. H.G. & .86 & 13 & 13 & 19.3 \\
\hline Average male & .66 & 11 & 11 & 38.3 \\
\hline Average female & .65 & 13 & 14 & 27.1 \\
\hline
\end{tabular}

Note-The VPEL score is given as the slope of the VPEL-versus-pitch function of each individual; GCT is the number of correctly identified parts-deleted line drawings; SPT is the number of correctly identified fragmented line drawings with added white noise; and EFT is the average time spent searching for a simple figure within the corresponding complex figure. (VPEL was measured at each of seven pitches; the individual VPEL values for each subject at each pitch that underlies the slopes shown here are given in Hudson, Li, \& Matin, 2000, Table 1.)

two, the other had five). The individual means for the EFT completion times are given in Table 1.

\section{Visually Perceived Eye Level Discrimination}

VPEL settings varied approximately linearly with the magnitude of the pitch of the visual field for each subject and the average of the subjects (all $r^{2}$ values $>.95$ ). There were individual differences in the slopes of the least-squares fit of VPEL as a function of physical pitch $\left(V^{\prime}\right)$, which ranged in magnitude from .33 to .86 , with a median of .65. The slope of the function relating settings of VPEL to physical pitch is given in Table 1 for each subject. The average of all the subjects' VPEL settings, along with average VPEL settings for one representative subject (J.H.), are given in Figure 4.

\section{Gestalt Completion Test and Snowy Pictures Test}

The subjects had no trouble finishing the GCT and the SPT within the 2 or $3 \mathrm{~min}$ (respectively) allotted. Nevertheless, all the subjects skipped at least one item, except Subject L.S., who gave a response for every item on the SPT. The median number of correct responses was 12 for the GCT and 13.5 for the SPT. All the subjects responded correctly on at least half of the items for which they made a response. The total number of correctly identified items for the GCT and SPT are given for each subject in Table 1. 


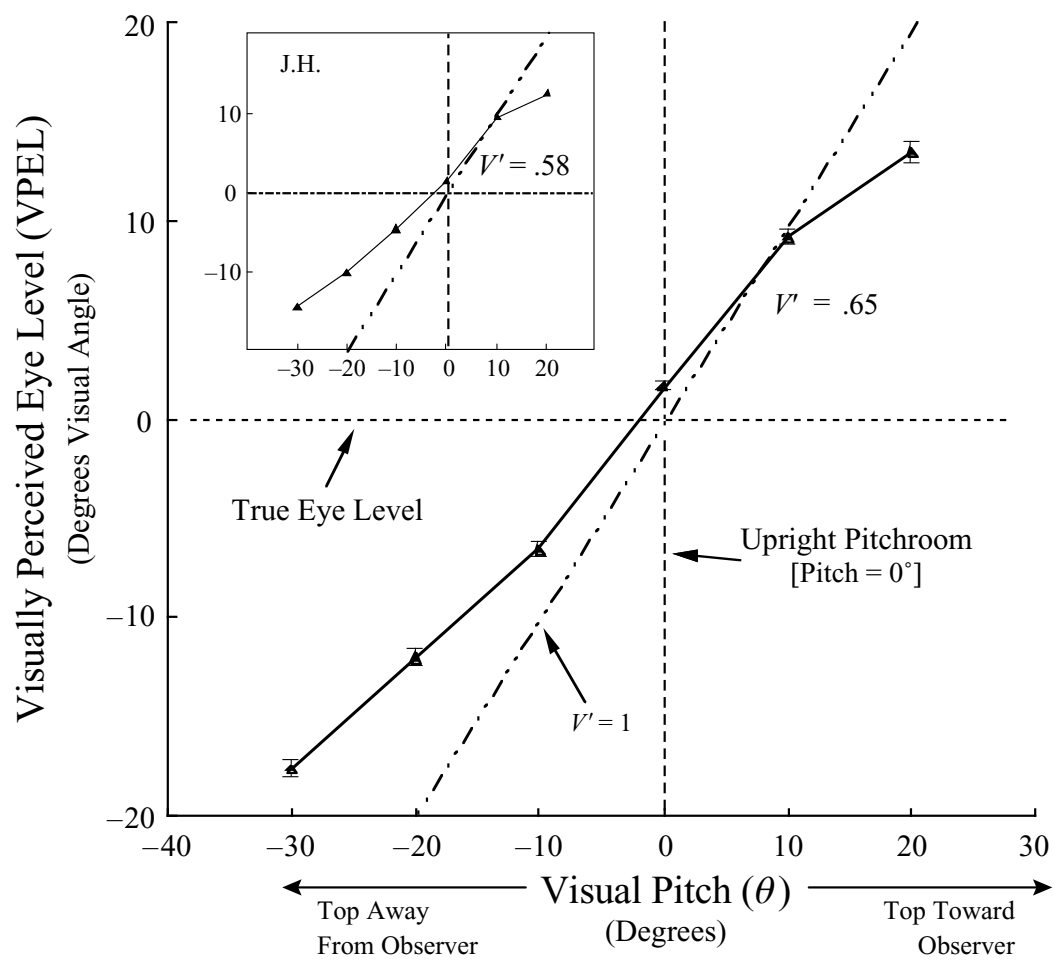

Figure 4. Mean settings of visually perceived eye level (VPEL) as a function of physical pitch for all the subjects and for a single representative subject (J.H.). The horizontal dashed line at VPEL $=0^{\circ}$ indicates veridical VPEL settings. The dashed line with a slope of 1 -indicating a VPEL-versus-pitch slope $\left(V^{\prime}\right)$ of 1 -indicates the location of the normal to the visual field. Error bars are standard errors of the mean VPEL settings.

\section{Relations Among Measures}

We first note that an increase in the number of correct items in the GCT and in the SPT corresponds to greater field independence, but an increase in the slope of the VPEL-versus-pitch function and an increase in search time in the EFT correspond to less field independence (greater field dependence). This leads to a problem for dealing with the correlations among these measures: Since it is desirable that a positive correlation correspond to measurements that reflect a trend for increased field independence for an individual in both measures, if we employ the values in Table 1 we must reverse the sign of the correlation that results from a calculation between the GCT and the VPEL-versus-pitch function, between the GCT and the EFT, and between the SPT and the VPEL-versus-pitch function, as well as between the SPT and the EFT; if we were to do otherwise, a negative correlation would result when the trends of the two measures both reflect increased field dependence for an individual. Thus, following calculation of these four correlations, the signs will be reported herein as the reverse of the value that was actually calculated from the values in Table 1. (Note that in order to accomplish the same end, the sign of the correlations between the GCT and the SPT and between the slope of the VPEL-versus-pitch function and the EFT will not be reversed from the sign calculated with the data in Table 1.)
When the convention in the previous paragraph was employed, all of the cognitive tests were significantly correlated positively with one another (see Figure 5A): The correlation between EFT completion times and the number correct for the GCT was $r(16)=+.73(p<.01)$. The correlation between the EFT and the number correct on the SPT was $r(16)=+.48(p<.05)$. The correlation between the numbers correct on the GCT and the SPT was $r(16)=+.71(p<.01)$. There were no significant correlations between VPEL slopes and the three cognitive tests: EFT completion times, $r(16)=+.09$; GCT scores, $r(16)=+.13$; or SPT scores, $r(16)=+.02$ (see Figure 5B).

\section{DISCUSSION}

\section{Lack of Commonality Between the VPEL and the EFT Tasks}

Although both the perception of eye level and the facility with which subjects identify embedded figures are dependent on the context provided by the prevailing visual field, the magnitude of the effect of the visual field on perception in the VPEL task is not related to the magnitude of the disturbing effect of the visual field on perception in the EFT (Figure 5B: the $r=+.09$ is not significant). ${ }^{3}$ This has implications for the concept of psychological dif- 
A
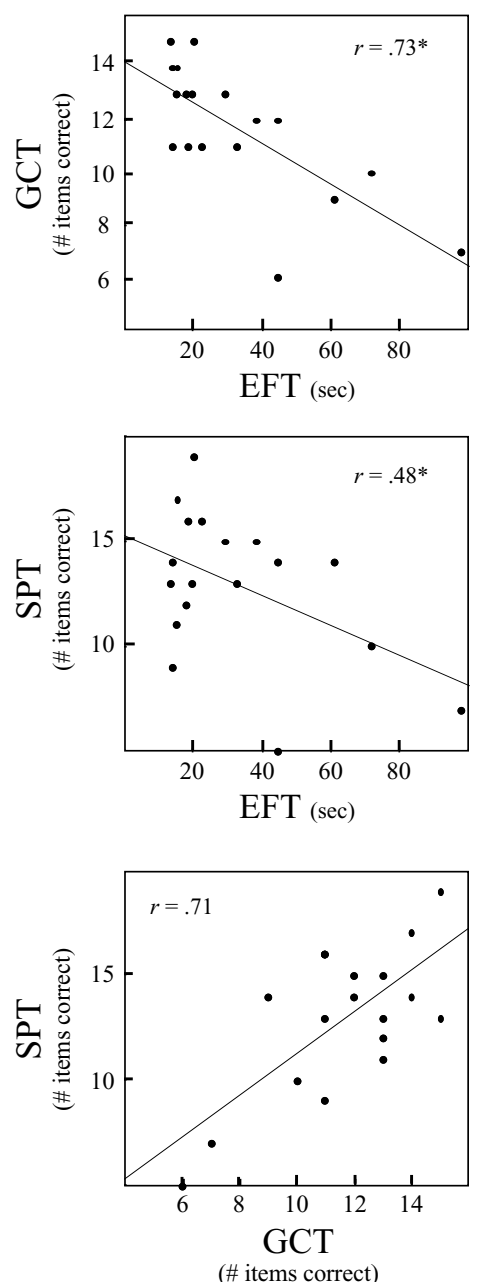

B
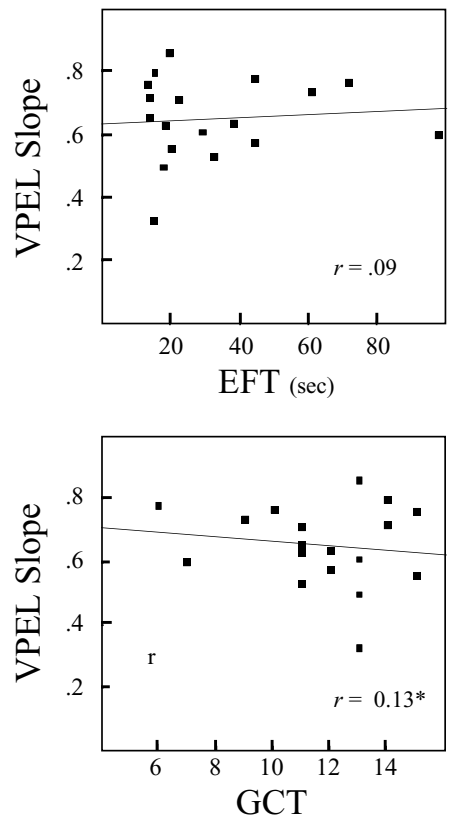

(\# items correct)

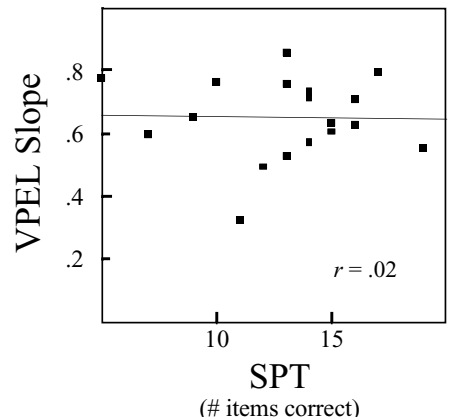

Figure 5. (A) Scatterplots of individual subjects' scores on the three cognitive tests: Gestalt Completion Test (GCT), Snowy Pictures Test (SPT), and Embedded Figures Test (EFT). (B) Scatterplots of the slopes of individual subjects' visually perceived eye level (VPEL) discriminations versus cognitive test scores (separately for each of the three tests). The three correlations in panel A are significant $(p<.01)$; the three correlations in panel $B$ are nonsignificant $(p>.1)$. In all plots, the line through the points is a least-squares best fit. *The signs of the asterisked values are reversed from the values calculated from the raw values in Table 1; see the text for a detailed rationale.

ferentiation, from which the concepts of field dependence and field independence have been derived. Psychological differentiation, measured by either EFT or RFT performance, has been supposed to provide an index of the extent to which internal or external referents determine behavior. For example, a subject with a relatively undifferentiated cognitive style is expected to be relatively more influenced by external than by internal sources of information in completing a task - regardless of whether the task requires differentiating between internally and externally derived information about the direction of gravity (VPV discrimination), perceiving the egocentric direction cor- responding to eye level (VPEL discrimination) in the face of visual inducing stimuli that might lead to nonveridical perceptions, or differentiating between the dominant figural groupings of a complex geometric figure and the internally held representation of a simple geometric figure (EFT). Thus, psychological differentiation has been considered to be a factor that mediates performance on cognitive tasks and on spatial tasks in which internal and external information would provide conflicting guides and would lead to different performances on a given task.

This assumption, that there is a processing style that determines how an individual solves such perceptual prob- 
lems, provides the basis for the conclusion that certain general personality characteristics are developed through chronic activation of a restricted set of field-dependent or field-independent perceptual-processing strategies. Personality characteristics relating to the application of internal/external referents in social situations as diverse as the use of interpersonal space (Witkin, 1977), reactions to the road/driver environment likely to produce an automobile accident (Mihal \& Barrett, 1976), and the weighting of others' opinions in conflict situations (Witkin, 1977; Witkin \& Goodenough, 1977) were therefore assumed to derive from these perceptual characteristics. Our results, however, are inconsistent with the generality of such a factor of cognitive restructuring in the processing of the egocentric perception of space.

Thus, the independence between EFT and VPEL in our results cannot be accounted for by a system employing a perceptual processing mechanism as portrayed in Figure 6A but requires at least two mechanisms (as, e.g., in Figure 6B). Whereas the weights, $\alpha$ and $\beta$, in Figure 6A contain only one degree of freedom $(\alpha+\beta=1)$, those in Figure 6B contain two degrees of freedom $\left(\alpha_{1}+\beta_{1}=1 ; \alpha_{2}+\beta_{2}=1\right)$. If the two degrees of freedom in Figure 6B- $\alpha_{1}$ and $\alpha_{2}$ (or $\beta_{1}$ and $\beta_{2}$ ) - are not correlated across individuals, performances on the EFT and VPEL tasks would also be predicted to be uncorrelated, in agreement with the results of the present experiments.

\section{Multiple-Factor Treatment}

In order to provide a more general basis for dealing with the relations among all of the different experimental mea- sures, a factor analysis of the correlations was carried out. For this purpose, in addition to the four experimental variables described in the Method section (VPEL, GCT, EFT, and SPT), a fifth variable that had been measured in these subjects was also included: This fifth variable provided a second egocentric spatial dimension, the perceived orientation of the inducing field; we refer to it as perceived visual pitch (PVP; see Hudson et al., 2000). PVP measurements of the orientation at which the pitchroom was perceived to be pitched for each of the six physical pitches were made employing a psychophysical matching procedure: In brief, for the PVP measurement, the subject instructed the experimenter to set the orientation of a small rectangular surface to match the perceived orientation of the pitchroom; the small matching surface was viewed against an erect wall located behind the seated subject; the pitchroom and matching surface were viewed successively on each trial. The relation between the slopes of the PVPversus-pitch and VPEL-versus-pitch functions measured in the pitchroom over the $-30^{\circ}$ to $+20^{\circ}$ range of pitches was described in a previous article in which further procedural details for the PVP task are presented (Hudson et al., 2000). Despite the fact that VPEL depended on the pitch of the pitchroom, that the mean VPEL grew with physical pitch with a slope of .65 (Figure 4), that PVP rose nearly linearly with physical pitch with a slope of .95 , and that the means of the VPEL and PVP settings at each pitch for each subject were strongly correlated $[r(118)=.89, p<$ $.001]$, the correlation between the slopes of the VPELversus-pitch and PVP-versus-pitch functions across subjects did not differ significantly from zero $[r(18)=-.10$,

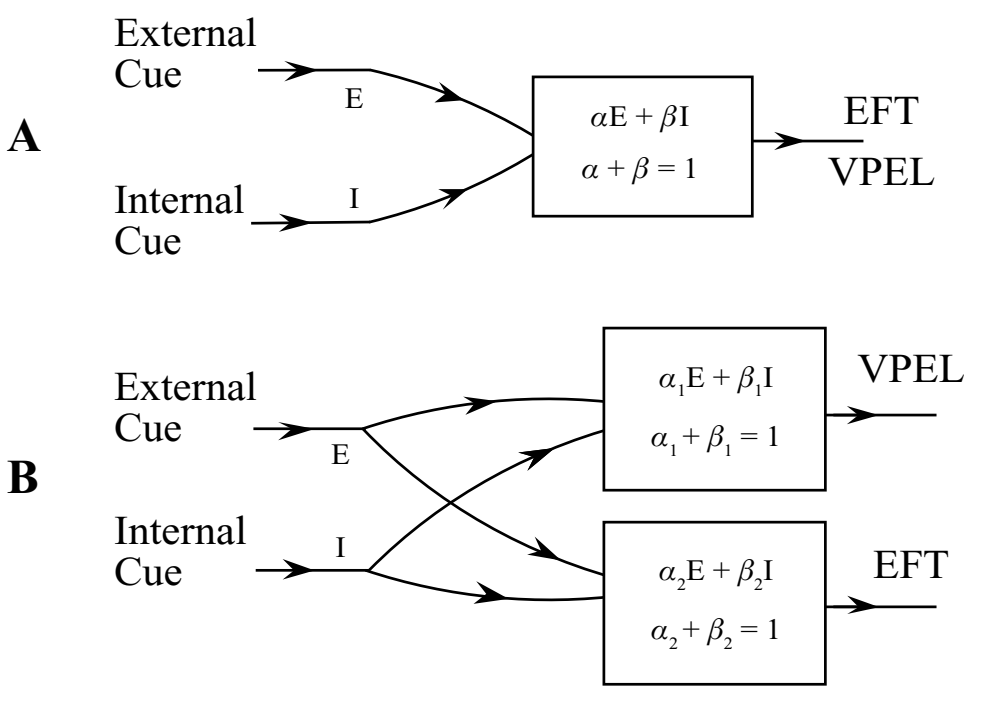

Figure 6. (A) Relationship of connections between sensory input and perceptual output, as suggested by previous models. It is assumed that $\alpha+\beta=1$. (B) Relationship of connections between sensory input and perceptual output suggested by the present results. The gains of the sensory input from internal and external cues to the visually perceived eye level (VPEL) discrimination $\left(\alpha_{2}\right.$ and $\beta_{2}$ weights) are independent of those for the same cues to the Embedded Figures Test (EFT; $\alpha_{1}$ and $\beta_{1}$ weights). It is assumed that $\alpha_{1}+\beta_{1}=1$ and that $\alpha_{2}+\beta_{2}=1$. 
$p>.1]$, and the correlations across individual subjects between the means of the VPEL and PVP settings for each magnitude of physical pitch were all nonsignificant (for all values, $p>.1$ ). Thus, although the VPEL and PVP settings appear to be determined by mechanisms that have a great deal in common for each individual, that relation is different for different individuals.

The correlations among the five experimental variables (VPEL, PVP, EFT, GCT, and SPT) are summarized in Table 2 . The set of 10 correlations was factor analyzed by means of a principal-components extraction, followed by an oblique rotation to an oblimin criterion (SPSS, Version 10, 2000). The pattern matrix in Table $3 \mathrm{~A}$ contains the oblique factor loadings of the five experimental variables on the three factors; the communalities involving the three orthogonal common factors resulting from the principal-components extraction are displayed in the diagonal cells of Table 2 . The relation between the three oblique factors and the five experimental variables are displayed as endpoints of the unit vectors on the surface of a sphere in Figure 7 (the other ends of the vectors converge at the center of the sphere) with the angular great circle distances between points obtained from the correlation between the two endpoints: the direction cosines in the normalized orthogonal factor matrix (variable-variable separation), the factor structure matrix (variable-factor separation), and the primary factor intercorrelation matrix (factor-factor separation). The intersections between the lighter solid lines represent the locations of the orthogonal factors resulting from an original orthotran rotation.

The loadings in Table $3 \mathrm{~A}$ provide a basis for a clear separation of the three factors (see Table 3B): The three cognitive tests (EFT, GCT, and SPT) are highly loaded on the first factor, Factor I, along with negligible loadings of VPEL and PVP. The high loading of VPEL stands alone on Factor II, with the loadings of the other four variables near zero, and the high loading of PVP stands alone on Factor III, with loadings of the other four variables nonsignificant. The cluster of three cognitive tests is thus clearly separated from both VPEL and PVP; in addition, VPEL and PVP are clearly separated from each other. These results of the factor analysis are, of course, consistent with the view that can be gained from inspecting the original

Table 2

Correlation Matrix

\begin{tabular}{lrrrrr}
\hline & VPEL & EFT & PVP & GCT & SPT \\
\hline VPEL & 1.000 & & & & \\
EFT & .090 & .980 & & & \\
PVP & -.100 & .070 & .999 & & \\
GCT & $.130^{*}$ & $.730^{*}$ & .290 & 1.000 & \\
SPT & $.020^{*}$ & $.480^{*}$ & .170 & .710 & .982 \\
\hline
\end{tabular}

Note-The off-diagonal cells contain the correlations among the five experimental variables. The diagonal cells contain the communalities from the principal-components extraction. VPEL, visually perceived eye level; EFT, Embedded Figures Test; PVP, perceived visual pitch; GCT, Gestalt Completion Test; SPT, Snowy Pictures Test. ${ }^{*}$ The signs of the asterisked values are reversed from the values calculated from the raw values in Table 1; see text for detailed rationale.
Table 3A Factor Pattern Matrix

\begin{tabular}{lrrr}
\hline & Factor I & Factor II & Factor III \\
\hline VPEL & .000 & 1.000 & -.003 \\
EFT & 1.000 & .017 & -.134 \\
PVP & .007 & -.005 & .998 \\
GCT & .962 & .062 & .122 \\
SPT & .995 & -.071 & .040 \\
\hline
\end{tabular}

Note-The values in the table are the oblique factor loadings of the five experimental variables. VPEL, visually perceived eye level; EFT, Embedded Figures Test; PVP, perceived visual pitch; GCT, Gestalt Completion Test; SPT, Snowy Pictures Test.

Table 3B

Primary Intercorrelations

\begin{tabular}{lrcc}
\multicolumn{4}{c}{ Primary Intercorrelations } \\
\hline & Factor I & Factor II & Factor III \\
\hline Factor I & 1.000 & & \\
Factor II & .092 & 1.000 & \\
Factor III & .195 & -.092 & 1.000 \\
\hline
\end{tabular}

Note-The values are correlations among the three oblique factors.

table of correlations in which the threefold separation is clear: The original correlations between VPEL and each of the three cognitive tests are not far from zero; the correlations between PVP and the three cognitive tests are small at best; the correlations between VPEL and PVP are not far from zero; the correlations among the three cognitive tests are substantial.

However, although at least three factors are required to account for the correlations among the five variables, as is shown by the analysis above, three are not sufficient. The need for additional dimension(s) arises wholly from the results with the single group of subjects in the present experiments: Figure 8 displays the angular distances between the five test variables that would separate them on a spherical surface such as that shown in Figure 7 if the great circle distances between them $(\Phi)$ were calculated from the measured correlation between the two variables as $\cos \Phi_{i j}=r_{i j}{ }^{4}$ Two of these distances (VPEL/SPT and PVP/SPT) are represented by dashed lines (see Figure 8), and the distance calculated from the correlations between the two variables for each of these two distances is shown in Table 4 as the "empirical distance." However, the distance from VPEL to SPT on the spherical surface is also constrained by the remainder of the set of great circle distances between VPEL, EFT, GCT, and EFT. Similarly the distance from PVP to SPT on the spherical surface is also constrained by the remainder of the set of great circle distances between PVP, EFT, GCT, and EFT. The distances determined by these latter constraints are listed in Table 4 as the "inferred distance." The difference between the empirical distances resulting from the correlation between the two correlated variables themselves (VPEL/SPT and PVP/SPT) and the inferred distance in each case is substantial - equal to $38^{\circ}$ and $36^{\circ}$, respectively. These differences are too large to be the result of unreliability in the psychophysical measurements in VPEL or PVP: The variability in the measurement of those variables is small: thus, for a given subject with 


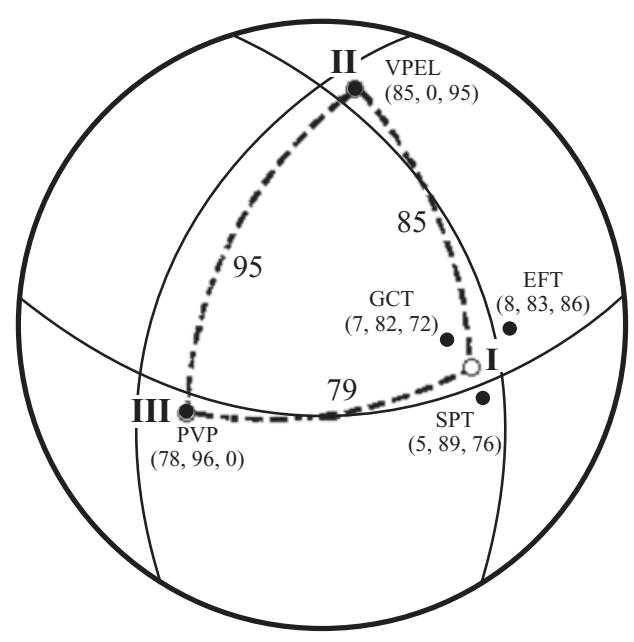

Figure 7. Three factors with origin at the center of the unit sphere and endpoints shown on the surface, along with the endpoints for the five experimental variables (all are normalized unit vectors). Unfilled circles are the oblique primary factors: I, II, and III, respectively. Filled circles are the experimental variables. The three numbers in parentheses next to each experimental variable are great circle distances (in degrees) from the variable to the three primary factors in the order I, II, III; single numbers along dashed lines representing arcs on the sphere are great circle distances between the oblique primary factors at the endpoints of the arc as angular values on the sphere. VPEL, visually perceived eye level; EFT, Embedded Figures Test; GCT, Gestalt Completion Test; SPT, Snowy Pictures Test; PVP, perceived visual pitch.

a given pitchroom orientation, the variability among four successive measurements at a given pitch is less than $1^{\circ}$ for VPEL and less than $3^{\circ}$ for PVP; the best-fitting slope for each subject involves six different orientations and is extremely stable for a given individual. In addition, the high correlations among the three cognitive tasks $(.71, .48$, and .73), along with their separation from VPEL and PVP, testify to their dependence on a common set of abilities that share little, if anything, with those involved in VPEL and PVP discrimination. Thus, the large differences between the empirical and the inferred distances in Table 4 tell us that three dimensions are not sufficient to adequately describe performance on these tasks. They indicate the presence of other factors that are central to performance on some of the experimental variables but that cannot be teased out as common factors with only five experimental variables. ${ }^{5}$

The present results can be combined with the classical results in which performances on EFT and the VPV discrimination have been reported to be significantly correlated (Witkin et al., 1954). ${ }^{6}$ In addition, significant correlations between VPEL and VPV have been reported from three different laboratories: Poquin et al. (1998), Guzy et al. (2003), and Shavit et al. (2004). These results are displayed in Figure 8 as add-ons to the results of the factor analysis of the correlations in Table 2 from the present study. Whatever the common aspect to EFT and VPV, the nonsignificant correlation between VPEL and EFT implies that it must be different from the aspect shared by
VPEL and VPV. ${ }^{7}$ Nevertheless, the VPEL/VPV correlations do share processing, as will be discussed below.

Certainly, the conjunction of the previous sets of results with the present set is not expected from a theory stating that disembedding tasks (including spatial tasks such as the VPEL, VPV, and PVP discriminations) are the result of a unitary mechanism. Clearly, not all spatial tasks involve a common processing mechanism. Whatever the basis for the link between the VPV and the EFT, it is not general for all spatial tasks. ${ }^{8}$ Thus, it is of considerable interest that the factorial description in Figure 8 is able to incorporate the threefold orthogonality between VPEL, PVP, and EFT in the present report and remain consistent with the previously reported positive relation between EFT and VPV.

\section{Relations Between the VPEL and VPV Discriminations}

The present results provide no support for a link between the internal processing leading to the VPEL discrimination and to the embedded figures performance or the two other tests of cognitive abilities that were highly correlated with the EFT. Nor was there any support for a link between the internal processing leading to the PVP discrimination, our second spatial dimension, and any of the three cognitive tests. However, as was noted above, significant correlations between VPEL and VPV have been reported from three different laboratories: Poquin et al. (1998), Guzy et al. (2003), and Shavit et al. (2004). ${ }^{9}$ Although these correlations again bring to mind consideration of the possibility of a contribution by cognitive restructuring, particularly in view of the previous support for such a basis for the VPV/EFT correlation, the present failure of VPEL and the signature measure of disembedding (EFT) to manifest any relation suggests that we look elsewhere for the basis for the VPV/VPEL correlations. The experiments from our laboratory (Shavit et al., 2004) suggest the combination of two sources of influence.

1. Shavit et al.'s (2004) VPV/VPEL correlations for 20 subjects were obtained with one-line and two-line inducers in the frontoparallel plane: two $70^{\circ}$-long, variably rolltilted lines $25^{\circ}$ eccentric on the left and right of the median plane..$^{10}$ The values of the correlations were similar to those measured with the square frames by Poquin et al. (1998) and Guzy et al. (2003). Thus, the +.45 correlation noted in note 9 was between the slope of the VPV-versus-rolltilt function measured with parallel two-line inducers and the slope of the VPEL-versus-roll-tilt function measured with bilaterally symmetrically oriented two-line inducers; this was the appropriate correlation because the two lines of the bilaterally symmetrically oriented two-line inducer are retinally identical to the two significant lines in the pitched frame that influence VPEL systematically. In addition, the VPV/VPEL correlation by the variably oriented right and left lines measured separately were $r=+.63$ and $r=-.37$, respectively (the negative correlation for the influences by the left line is a consequence of the bilateral symmetry of the influence on VPEL, coupled with the identity of influence on VPV). ${ }^{11}$ 


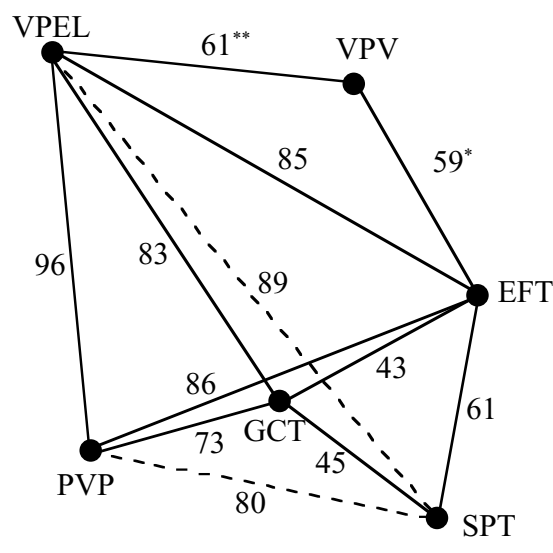

Figure 8. Flattened spherical surface showing angular distances between variables. Numbers next to lines connecting variables indicate the great circle distances, $\Phi$, between those variables on a sphere, as calculated from the correlation matrix (the correlation between the two variables at the ends of the connecting line equals the cosine of $\Phi$ ). Dashed lines indicate relationships that cannot be consistently represented on the surface of the sphere, given the pattern of interrelationships with other variables. The inconsistency of the data with a spherical representation is due entirely to the data collected in the present experiment, and not to data included in this figure that were obtained in other laboratories. *The angular distance shown between the Embedded Figures Test (EFT) and visually perceived vertical (VPV) is calculated from the average of the correlation values for males and females for the erect case in Witkin's results (see note 8). **The angular distance shown between visually perceived eye level (VPEL) and VPV is calculated from the average of five values: Poquin, OhImann, and Barraud (1998; $r=+.67)$; Guzy, Cohen, and Ebenholtz (2003; $r=+.37 ; r=$ $+.29)$; Shavit, Li, Semanek, and Matin (2004; $r=+.63$ for the right one-line stimulus; $r=+.45$ for the parallel two-line stimulus). Including these correlations with the 10 correlations from the present study places no additional constraints on the threedimensional fit obtained from the $\mathbf{1 0}$ correlations in Table 2.

Earlier work (Matin \& Li 1994b, 1995) had shown that a unidimensional description does not suffice for representing the influences of line orientation on both VPV and VPEL even when the identical distal stimulus lines are employed for both the VPV and the corresponding VPEL measurements; two linearly independent orientationsensitive perceptual-processing dimensions are required to explain the visual component of the results generated with one-line and two-line inducing stimuli. In Shavit et al.'s (2004) experiments, each of the orientations of the roll-tilted inducer line was employed for both VPV and VPEL measurements. Thus, the correlated measurements for both discriminations were made with the inducing line at the identical retinal orientations. In addition, fMRI measurements (Matin, Li, Hudson, \& Hirsch, 2001) had shown that the processing of the two discriminations is controlled in two different regions of the cerebral cortex; left area $19(\mathrm{GOM})$ is involved in the processing of the two-line stimulus whose orientation systematically influences VPV but not VPEL, whereas right area 40 (LPi) and left area $7(\mathrm{PCu})$ are involved in the processing of the twoline stimulus whose orientation systematically influences VPEL but not VPV.
The combination of results described here suggests the following two-stage model as a basis for the two discriminations. (1) Both discriminations are processed by the same orientation-sensitive neural units in the input stage of area $\mathrm{V} 1$, primary visual cortex, and accounts for the common variance in the VPV/VPEL correlation. (2) The subsequent stages of processing are carried out separately by different higher order processes in different cortical regions. Such a model for the processing of VPV and VPELcommon lower level processing and separate higher level processing in the cerebral cortex-accounts well for the original requirement of two higher order mechanisms to account for the basic results with one-line and two-line stimuli with VPV and VPEL discriminations (Matin \& Li, 1992a, 1992b, 1994a, 1994b), as well as for the more recent correlations between the two discriminations for the experiments with one-line and two-line inducers and for the experiments with the frame, without invoking a still higher common level of processing that involves cognitive restructuring (Shavit et al., 2004).

2. However, there is a second possible basis for the correlations between VPV and VPEL measures: The relation between VPEL and the orientation of the one-line or twoline inducers is nearly linear, as is the relation between VPV and the orientation of the one-line or two-line inducers (Li \& Matin, 2005a, 2005c; Matin \& Li, 1992a, 1992b, 1994a, 1994b). These near-linear relations have been well fitted by straight lines with slopes that are invariably less than the slope of 1.00 corresponding to the slope for which there is a 1:1 relation between the discrimination measure-VPEL or VPV - and line orientation. The slope of the best-fit straight line has been interpreted as a measure of sensitivity to the visual induction stimulus since the original discovery of the influence of visual pitch on VPEL in the fully illuminated and well-structured pitchroom (Matin \& Fox, 1986, 1989). Correlations between the $y$-intercept and dark values have invariably been considerable, and both have been interpreted as measures of the influence of the body-referenced mechanism alone, although the dark value is measured in complete darkness and the $y$-intercept is measured in the presence of a visual inducing stimulus (Li et al., 2001; Matin \& Fox, 1989; Matin \& Li, 1994a). Recent work (Shavit et al., 2004) has also measured large correlations between the dark values for VPV and VPEL, between the $y$-intercepts for VPV and VPEL, and between the dark value and $y$-intercept in each

Table 4

Discrepant Spherical Distances

\begin{tabular}{lccc}
\hline & Empirical Distance & Inferred Distance & Difference \\
\hline VPEL/SPT & $89^{\circ}$ & $127^{\circ}$ & $38^{\circ}$ \\
$\mathrm{PVP} / \mathrm{SPT}$ & $80^{\circ}$ & $116^{\circ}$ & $36^{\circ}$ \\
\hline
\end{tabular}

Note-Both distances for each pair of experimental variables assume the three-dimensional spherical representation shown in Figure 7. The "empirical distances" are values calculated directly as the arccosines of the correlations between the two experimental measures. The "inferred distances" are values calculated from the constraints imposed by the experimental measures other than the two endpoints. VPEL, visually perceived eye level; SPT, Snowy Pictures Test; PVP, perceived visual pitch. 
case. These suggest that although VPV and VPEL are settings in entirely different dimensions of visual space, there is considerable commonality in the operation of the bodyreferenced mechanism in both. This commonality could contribute to the correlations measured between VPV and VPEL under induction by the one-line and two-line stimulus (see also Ohlmann \& Marendaz, 1991).

Thus, whereas the first explanation represents an attempt to explain the VPV/VPEL correlations wholly in terms of the visual inducing stimulus, the second explanation relies wholly on the body-referenced mechanism. Undoubtedly the truth lies in their combination, and it is reasonable to believe that the model in Figure 6B, which makes use of both for VPEL, will apply to VPV also, although the weights will differ for the two discriminations.

\section{Further Considerations Regarding Field Dependence/Independence}

In the original descriptions of a commonality in the processing of the VPV discrimination and EFT performance (Witkin, 1950; Witkin et al., 1954), three separate experiments were reported. The male/female difference in those experiments - significant VPV/EFT correlations for males and low, nonsignificant correlations for females - has often been commented on, although it has not always been replicated (see note 6). We note two matters related to these differences that point to problems with the viewpoint that treats them as reflecting a difference in field dependence. (1) Employing the magnitude of the influence of a roll-tilted visual field as the measure of field dependence, Witkin's original results clearly indicate that females are more field dependent. However, although not necessary, more field-dependent individuals would be expected to exhibit larger correlations between RFT and EFT and not smaller ones - certainly not insignificant ones, as in Witkin's original reports. (2) Two sets of experiments report that the gender difference on the EFT vanishes with practice (Chance \& Goldstein, 1971; Goldstein \& Chance, 1965; see note 6). Such a result is certainly not what one would expect from a stable personality trait, as FDI has been considered to be. It also seems likely that today, women may have had their "practice" in the real world, and the gender difference might be even further diminished or gone.

Witkin appears to have begun a reformulation of his conception of psychological differentiation during the preparation of his last book, published posthumously (Witkin \& Goodenough, 1981). In that book, he describes several major revisions of his theory through the years. Of special interest in the present context is this statement:

It now seems possible that what we earlier designated an articulated-global field approach consists of two separate though related functions: reliance on vestibular or visualfield referents and cognitive restructuring. In this conception we revert to our very earliest view of performance on tests of perception of the upright, and put in question our subsequently held view that at the essence of performance in both tests of perception of the upright and tests such as the EFT is competence in disembedding. (p. 47)
These statements are roughly consistent with the view represented in Figure $6 \mathrm{~B}$, in that "reliance on vestibular or visual-field referents" maps onto our use of bodyreferenced mechanism or visual stimulation as internal or external cues, respectively. However, they leave open the question of the basis for their earlier results relating cognitive restructuring and the relative reliance on vestibular or visual field referents. It was the attempt to explain those empirical results that led to the concept of disembedding and the assumption of a characteristic processing style for the solution of a range of perceptual and cognitive problems involving disembedding.

Ten years later, Wapner and Demick wrote a summary chapter entitled "Some Open Research Problems on Field Dependence-Independence: Theory and Methodology" in the book Field Dependence-Independence, which they edited (Wapner \& Demick, 1991). The book was the publication of a symposium held in honor of Herman Witkin, who had died in 1979; the conference was also opened by Wapner, who had collaborated with Witkin in some research related to FDI. In this last chapter, Wapner and Demick state that

during the conference on which this volume is based, a number of questions surfaced regarding the theoretical conceptualization underlying performance on the RFT and the EFT. ... The oft heard question was "What is the nature of the field dependence-independence construct? Is it one construct or two?" Visual versus postural conflict is presumably reflected in RFT performance; restructuring ability is presumably reflected in EFT performance. Are these two separate components of FDI, that is, should they be treated as distinct processes with common variance. . . . Thus, one important unresolved question is: What general research strategies can be used to attack the general problem of the nature of the processes underlying the two main test situations? (pp. 403-404)

Wapner and Demick's (1991) summary was published 37 years after the original publication of the correlations between RFT and EFT (see note 6) that have been the fundamental base to the subsequent mountain of work on the dependence-independence story during the intervening years. The last sentence reraises the very same question that Witkin's original work in 1954 raised. It continues to be of interest.

At this time, the view that FDI is responsible for the early findings of a relation between the RFT and the EFT appears to retain its position as the dominant one, despite these comments by Witkin and Goodenough (1981) and Wapner and Demick (1991).

Our own belief is that the individual subject's stability across tasks centered on the perception of the vertical in the RFT measurements that Witkin and Asch (1948) originally discovered, a stability that held at one level for a given individual but at different levels for different individuals, is of extreme importance. This description of stability in egocentric space perception was being described for the first time in the 1948 reports; such stability also holds for VPEL and, further, holds over many years. However, rather than pointing to control by a trait involving cogni- 
tive restructuring or a cognitive style based on the bipolar trait of FDI, it now appears that it points to the hardwired and relatively immutable mechanism based on the relative weighting of influences from the visual field and from the body-referenced mechanism, a weighting that varies over a large range from individual to individual. Indeed, cognitive restructuring seems particularly unlikely for both VPV and VPEL: The effects of visual induction in both the VPV and the VPEL discriminations are immediate and do not involve cognitive searches such as those involved in the three cognitive tests examined in the present experiments, and detailed knowledge of the influence of the effects of spatial induction-even repeated exposure and repeated back-and-forth viewing of the peculiar aspects of the inducing scene from within and outside the illusioninducing visual environment, with attempts at analysis by the subject - has no significant influence on an individual's VPV or VPEL perceptual response.

\section{REFERENCES}

Annett, M. (1992). Spatial ability in subgroups of left- and righthanders. British Journal of Psychology, 83, 495-515.

Asch, S. E., \& WitKIN, H. A. (1948). Studies in space orientation: II. Perception of the upright with displaced visual fields and with body tilted. Journal of Experimental Psychology, 38, 455-477.

Bailey, W., Hustmyer, F., \& Kristofferson, A. (1961). Alcoholism, brain damage, and perceptual dependence. Quarterly Journal of Studies on Alcohol, 22, 377-393.

Bennett, D. H. (1956). Perception of the upright in relation to body image. British Journal of Mental Science, 102, 487-506.

Brosnan, M. J. (1998). Spatial ability in children's play with Lego blocks. Perceptual \& Motor Skills, 87, 19-28.

Chance, J. E., \& Goldstein, A. G. (1971). Internal-external control of reinforcement and embedded-figures performance. Perception \& Psychophysics, 9, 33-34.

Crandall, V. J., \& Sinkeldam, C. (1964). Children's dependent and achievement behaviors in social situations and their perceptual field dependence. Journal of Personality, 32, 1-22.

Ebenholtz, S. M. (1977). Determinants of the rod and frame effect: The role of retinal size. Perception \& Psychophysics, 22, 531-538.

Ebenholtz, S. M., \& Benzschawel, T. L. (1977). The rod and frame effect and induced head tilt as a function of observation distance. Perception \& Psychophysics, 22, 491-496.

Ekstrom, R. B., French, J. W., Harman, H. H., \& Derman, D. (1976). Manual for kit of factor-referenced cognitive tests 1976. Princeton, NJ: Educational Testing Service.

Freedman, R. J., \& Rovegno, L. (1981). Ocular dominance, cognitive strategy, and sex differences in spatial ability. Perceptual \& Motor Skills, 52, 651-654.

Geiringer, E. R., \& Hyde, J. S. (1976). Sex differences on Piaget's water-level task: Spatial ability incognito. Perceptual \& Motor Skills, 42, 1323-1328.

Goldstein, A. G., \& Chance, J. E. (1965). Effects of practice on sexrelated differences in performance on Embedded Figures. Psychonomic Science, 3, 361-362.

Gottschaldt, K. (1926). [Gestalt factors and repetition]. Psychologische Forschung, 8, 261-317. [English translation and condensation in W. D. Ellis (1958). A source book of gestalt psychology (pp. 109122). London: Routledge \& Kegan Paul.]

Guzy, L. T., Cohen, M. M., \& Ebenholtz, S. M. (2003). Field dependence with pitched, rolled, and yawed visual frame effects. In J. Andre, D. A. Owens, \& L. O. Harvey, Jr. (Eds.), Visual perception: The influence of H. W. Leibowitz (pp. 125-141). Washington, DC: American Psychological Association.

Hall, C. W., Gregory, G., Billinger, E., \& Fisher, T. (1988). Field independence and simultaneous processing in preschool children. Perceptual \& Motor Skills, 66, 891-897.
Harley, J. P., Kalish, D. I., \& Silverman, A. J. (1974). Eye movements and sex differences in field articulation. Perceptual \& Motor Skills, 38, 615-622.

HowarD, I. P. (1986). The perception of posture, self motion, and the visual vertical. In K. R. Boff, L. Kaufman, \& J. P. Thomas (Eds.), Handbook of perception and human performance: Vol. I. Sensory processes and perception (pp. 18.1-18.62). New York: Wiley.

HowARD, I. P., \& TEMPLETON, W. B. (1966). Human spatial orientation. New York: Wiley.

Hudson, T. E., Li, W., \& Matin, L. (2000). Independence of visually perceived eye level (VPEL) and perceived visual pitch (PVP). Vision Research, 40, 2605-2619.

KARP, S. A. (1963). Field dependence and overcoming embeddedness. Journal of Consulting Psychology, 27, 294-302.

Karp, S. A., Poster, D. C., \& Goodman, A. (1963). Differentiation in alcoholic women. Journal of Personality, 31, 386-393.

Li, W., Dallal, N., \& Matin, L. (2001). Influences of visual pitch and visual yaw on visually perceived eye level (VPEL) and straight ahead (VPSA) for erect and rolled-to-horizontal observers. Vision Research, 41, 2873-2894.

LI, W., \& MATIN, L. (1996). Visually perceived eye level is influenced identically by lines from erect and pitched planes. Perception, 25, 831-852.

LI, W., \& Matin, L. (1998). Change in visually perceived eye level without change in perceived pitch. Perception, 27, 553-572.

Li, W., \& Matin, L. (2005a). The rod-and-frame effect: The whole is less than the sum of its parts. Perception, 34, 699-716.

LI, W., \& Matin, L. (2005b). Two wrongs make a right: Linear increase of accuracy of visually-guided manual pointing, reaching, and heightmatching with increase in hand-to-body distance. Vision Research, 45, 533-550.

LI, W., \& Matin, L. (2005c). Visually perceived vertical (VPV): Induced changes in orientation by 1-line and 2-line roll-tilted and pitched visual fields. Vision Research, 45, 2037-2057.

Linn, M. C., \& Peterson, A. C. (1985). Emergence and characterisation of sex differences in spatial ability: A meta-analysis. Child Development, 56, 1479-1498.

Matin, L., \& AdKins, D. C. (1954). A second-order factor analysis of reasoning abilities. Psychometrika, 19, 71-78.

Matin, L., \& Fox, C. F. (1986). Perceived eye level: Elevation jointly determined by visual pitch, EEPI, and gravity. Investigative Ophthalmology \& Visual Science, 27(Suppl.), 333.

Matin, L., \& Fox, C. F. (1989). Perceived eye level and perceived elevation of objects: Linearly additive influences from visual field pitch and from gravity. Vision Research, 29, 315-324.

Matin, L., \& LI, W. (1992a). Mislocalizations of visual elevation and visual vertical induced by visual pitch: The great circle model. In B. Cohen, D. L. Tomko, \& F. E. Guedry, Sensing and controlling motion: Vestibular and sensorimotor function (Annals of the New York Academy of Sciences, Vol. 656, pp. 242-265). New York: New York Academy of Sciences.

Matin, L., \& LI, W. (1992b). Visually perceived eye level: Changes induced by a pitched-from-vertical 2-line visual field. Journal of Experimental Psychology: Human Perception \& Performance, 18, 257-289.

Matin, L., \& LI, W. (1994a). The influence of the orientation of a stationary single line in darkness on the visual perception of eye level. Vision Research, 34, 311-330.

Matin, L., \& LI, W. (1994b). Mirror symmetry and parallelism: Two opposite rules for the identity transform in space perception and their unified treatment in the great circle model. Spatial Vision, 8, 469-489.

Matin, L., \& LI, W. (1994c). Spatial summation among coextensive and parallel line segments across wide separations $\left(50^{\circ}\right)$ : Egocentric localization and the great circle model. Vision Research, 34, 2577-2598.

Matin, L., \& Li, W. (1995). Multimodal basis for egocentric spatial localization and orientation. Journal of Vestibular Research, 5, 499-518.

Matin, L., \& LI, W. (1999). Averaging and summation of influences on visually perceived eye level between two long lines differing in pitch or roll-tilt. Vision Research, 39, 307-329.

Matin, L., \& LI, W. (2000). Linear combinations of signals from two lines of the same or different orientations. Vision Research, 40, 517-527.

Matin, L., \& LI, W. (2001). Neural model for processing the influence of visual orientation on visually perceived eye level (VPEL). Vision Research, 41, 2845-2872. 
Matin, L., Li, W., Hudson, T., \& Hirsch, J. (2001). Perceptions of elevation and orientation: From the stimulus basis to the cerebral cortex. Journal of Vision, 1, 195a.

Minal, W. L., \& Barrett, G. V. (1976). Individual differences in perceptual information processing and their relation to automobile accident involvement. Journal of Applied Psychology, 61, 229-233.

Morant, R. B., \& Beller, H. K. (1965). Adaptation to prismatically rotated visual fields. Science, $\mathbf{1 4 8 , 5 3 0 - 5 3 1 .}$

NAYLOR, G. F. (1965). Subjective judgments of the vertical: An estimate of their nature and consistency. Australian Journal of Psychology, 17, 213-216.

O’Donnell, J. P., Paulsen, K. A., \& McGann, J. D. (1978). Matching familiar figures test: A unidimensional measure of reflectionimpulsivity? Perceptual \& Motor Skills, 47, 1247-1253.

Ohlmann, T., \& Marendaz, C. (1991). Vicarious processes involved in selection/control of frames of reference and spatial aspects of field dependence-independence. In S. Wapner \& J. Demick (Eds.), Field dependence-independence: Cognitive style across the life span (pp. 105-129). Hillsdale, NJ: Erlbaum.

Petrusic, W. M., Varro, L., \& Jamieson, D. G. (1978). Mental rotation validation of two spatial ability tests. Psychological Research, 40, 139-148

Pizzamiglio, L., \& Zoccolotti, P. (1981). Sex and cognitive influence on visual hemifield superiority for face and letter recognition. Cortex, 17, 215-226.

Poquin, D., Ohlmann, T., \& Barraud, P. A. (1998). Isotropic visual field effect on spatial orientation and egocentric localization. Spatial Vision, 11, 261-278.

Post, R. B., Teague, J. M., Welch, R. B., \& Hudson, T. E. (2003). Visually perceived eye level with reversible pitch stimuli: Implications for the great circle and implicit surface models. Perception, 32, 1073-1092.

Post, R. B., \& Welch, R. B. (1996). The role of retinal versus perceived size in the effects of pitched displays on visually perceived eye level. Perception, 25, 853-859.

Post, R. B., Welch, R. B., \& Clark, V. D. (2000). Why do pitched horizontal lines have such a small effect on visually perceived eye level? Perception \& Psychophysics, 62, 726-734.

Rhodes, R. J., CARR, J. E., \& JURJI, E. D. (1968). Interpersonal differentiation and perceptual field differentiation. Perceptual \& Motor Skills, 27, 172-174.

Shade, B. J. (1984). Field dependency: Cognitive style or perceptual skill? Perceptual \& Motor Skills, 58, 991-995.

SHAFFER, D. R. (2000). Social and personality development (4th ed.). New York: Wadsworth/Thomson Learning.

Shavit, A. Y., LI, W., Semanek, D., \& Matin, L. (2004). Individual differences in sensitivity to induction-by-line: Covariation between perceived elevation (VPEL) and perceived vertical (VPV) [Abstract]. Journal of Vision, 4, 383a. Available at journalofvision.org/4/8/383/, doi:10.1167/4.8.383.

Sherman, J. (1967). Problem of sex differences in space perception and aspects of intellectual functioning. Psychological Review, 74, 290299.

Stoper, A. E., \& Cohen, M. M. (1989). Effect of structured visual environments on apparent eye level. Perception \& Psychophysics, 46 469-475.

Stoper, A. E., \& Cohen, M. M. (1991). Optical, gravitational and kinesthetic determinants of judged eye level. In S. R. Ellis \& M. K. Kaiser (Eds.), Pictorial communication in virtual and real environments (pp. 390-403). London: Taylor \& Francis.

Street, R. F. (1972). A Gestalt Completion Test (Teachers College, Columbia University. Contributions to Education, no. 481). New York: AMS Press. (Original work published 1931)

Thurstone, L. L. (1944). A factorial study of perception. Chicago: University of Chicago Press.

Thurstone, L. L. (1947). Multiple-factor analysis. Chicago: University of Chicago Press.

WAPNER, S., \& DEMICK, J. (1991). Some open research problems on field dependence-independence: Theory and methodology. In S. Wapner \& J. Demick (Eds.), Field dependence-independence: Cognitive style across the life span (pp. 401-429). Hillsdale, NJ: Erlbaum.
WEINER, M. (1955). Effects of training in space orientation on perception of the upright. Journal of Experimental Psychology, 49, 367-373.

Wenderoth, P. (1982). Fine's frame and perceived tilt. Perceptual \& Motor Skills, 54, 763-766.

Williams, W. N., LaPointe, L. L., \& Riski, J. E. (1974). Lingual discrimination of angular changes of a grooved line by normal subjects. Perceptual \& Motor Skills, 38, 579-582.

WitKIn, H. A. (1949a). Perception of body position and of the position of the visual field. Psychological Monographs: General \& Applied, 63, 1-46.

WitKIN, H. A. (1949b). Sex differences in perception. Transactions of the New York Academy of Sciences, 12, 22-26.

WiTKIN, H. A. (1950). Individual differences in ease of perception of embedded figures. Journal of Personality, 19, 1-15.

WITKIN, H. A. (1977). Cognitive styles in personal and cultural adaptation (Heinz Werner Lecture Series, Vol. II). Worcester, MA: Clark University Press.

WitKin, H. A., \& Asch, S. E. (1948). Studies in space orientation: IV. Further experiments on perception of the upright with displaced visual fields. Journal of Experimental Psychology, 38, 762-782.

Witkin, H. A., Dyk, R. B., Faterson, H. F., Goodenough, D. R., \& KARP, S. A. (1962). Psychological differentiation. New York: Wiley.

Witkin, H. A., \& Goodenough, D. R. (1977). Field dependence and interpersonal behavior. Psychological Bulletin, 84, 661-688.

Witkin, H. A., \& GoOdEnough, D. R. (1981). Cognitive styles, essence and origins: Field dependence and field independence (Psychological Issues, Monograph 51). New York: International Universities Press.

WitKin, H. A., KarP, S. A., \& Goodenough, D. R. (1959). Dependence in alcoholics. Quarterly Journal of Studies on Alcohol, 20, 493-504.

Witkin, H. A., Lewis, H. B., Hertzman, M., Machover, K., MeissNER, P. B., \& WAPNER, S. (1954). Personality through perception. New York: Harper.

Witkin, H. A., Oltman, P. K., Raskin, E., \& Karp, S. A. (1971). A manual for the Embedded Figures Test. Palo Alto, CA: Consulting Psychologists Press.

\section{NOTES}

1. In the present context, the extent of differentiation refers to the extent to which one psychologically distinguishes, or differentiates, events perceived to originate within the organism (internal events) and those perceived to originate from the environment (external events).

2. We have employed the term body-referenced mechanism to include nonvisual or extraretinal inputs, including eye position and head position information, and those resulting from gravity's action on the body and from somesthetic stimulation, and we believe that this usage is consistent with the internal/external usage that Witkin employed as a basis for separating field-dependent from field-independent individuals.

3. The RFT/EFT correlations in the Witkin et al. (1954) work involved average values of the deviations of VPV from true vertical measured at two roll tilts of the inducing field $\left( \pm 28^{\circ}\right)$ to provide a measure of the influence of the visual field (see also note 6), whereas the present correlations involving VPEL employed the first derivative (the slope) of the psychophysical settings with respect to the physical variable (pitch). It is possible that the different measures play some role in the different outcomes. The reasons for our choice of the slope measure are the following. (1) A measure based on six stimulus values is inherently more reliable than a measure based on only one. (2) The slope of the VPEL-versus-pitch function is a measure of sensitivity to variation in the inducing stimulus (i.e., the first derivative of the function), as are the slopes of nearly all psychometric functions. (3) Over the range of pitches employed, the slope is nearly linear, making a linear best fit the simplest means of evaluating the function. (4) The slope is a bias-free measure in which the magnitude of displacement of the center value from true vertical plays no greater role than the magnitude of any of the other settings (the constant error is taken out of the picture). Thus, biases in settings of VPEL due to different criteria employed by individual subjects are essentially eliminated; such bias is essentially captured by the $y$-intercept of the linear best fit, a parameter that is independent of the slope. (5) The bias of the $y$-intercept of the linear fit is highly correlated (across subjects) with the value of the VPEL setting in total darkness in the absence 
of the inducing stimulus and, thus, is likely to reflect individual differences in criterion; the criterion itself is heavily influenced by extraretinal influences, largely arising from the body-referenced mechanism (see note 2). Since the interest here is in the susceptibility to visual induction, the slope of the function (i.e., the gain) provides a more appropriate, as well as a more reliable, measure.

4. Whereas each of the distances shown on Figure 8 between the experimental variables is equal to the cosine of the linear productmoment correlation between the two items at the endpoints of the line, the distance between a pair of experimental variables in Figure 7 is determined by the three-orthogonal-common-factor communalities (diagonal in Table 2) and the fact that placement of the experimental variable was set by the distances between the experimental variable and the three obliquely related factors.

5. Although it has become common to allow the extraction of common factors only with eigenvalues above 1 (as is true for the three factors here), with only five experimental variables, at most only two linearly independent common factors can be determined from the correlation matrix (Thurstone, 1944; 1947, p. 294). Our justification for extracting as many as three factors here was based on three matters. One of them was the apparent need for more than three common factors, as indicated in the text above. The second was the fact that the first two principal components account for only $69.5 \%$ of the total variance in the correlation table; adding the third principal component increases total variance explained to a more respectable $86.5 \%$. The third reason is that given the near-orthogonality between VPEL and PVP, the near-orthogonality between VPEL and each of the three cognitive tests, and the small correlations between PVP and the cognitive tests, extracting only two factors would lead to a result in which at least two of the experimental variables that are themselves not significantly correlated are required to have substantial loadings on the same factor; furthermore, one of these two factors needs to be bipolar. This only muddies the picture. It is worth noting that although it is possible to place a vector at a point on a great circle between two of the tests that are uncorrelated (e.g., on the circle that separates VPEL from SPT by $89^{\circ}$ in the representation in Figure 8), whether such a location would reasonably be considered as a location for a meaningful factor would depend on the correlation structure in a larger battery of tests. But as was noted above, it would be a peculiar sort of factor, since VPEL and EFT are statistically independent from each other. Certainly it would not be a unitary factor in the usual sense of that term, and although some locations for the vector on the great circle would allow the "factor" to contain a negative loading on one of the tests and a positive loading on the other, and although bipolar factors have been employed to stretch across angular distances greater than $90^{\circ}$ in both first- and second-order multiple-factor analyses (Matin \& Adkins, 1954; Thurstone, 1947), having the possibility of a common factor that contains reliably uncorrelated tests (the present case) is not reasonable.

6. Witkin et al. (1954) reported correlations between performances on the RFT and EFT measures for 46 male subjects at each of three body/frame orientation combinations: $r=+.47$ for the body roll tilted $28^{\circ}$ left or right with the frame roll tilted $28^{\circ}$ to the same side as the body; $r=+.43$ with the same frame tilts but with the body tilted $28^{\circ}$ to the opposite side of the body; and $r=+.76$ with the body erect and the frame at $\pm 28^{\circ}$. For 45 female subjects, the three correlations were: $+.03,+.22$, and +.26 . All three of the correlations for the male subjects were significant at the .01 level; none of the correlations for the female subjects was significant.

As was described in the Results section above, in the present case there were no significant correlations between VPEL and EFT. However, although the seven degrees of freedom is too small to allow us to draw broad conclusions, there were no significant correlations between EFT scores and $V^{\prime}$ values analyzed separately for female and male subjects: Females' $V^{\prime}$ and EFT scores had a correlation of $r(7)=-.09(p>.1)$, and the results for males also possessed a correlation of $r(7)=-.09$ $(p>.1)$. This is in contrast to the relation between EFT and GCT, for example, which showed high and significant correlations when male and female subjects were analyzed separately $(r=+.67, p<.05$, and $r=$ $+.89, p<.01$, respectively). (In a further search for significance, we found no correlation between $V^{\prime}$ and EFT scores when field-dependent subjects were separated from field-independent subjects $[r(7)=-.14$, $p>.1$, and $r(7)=.02, p>.1$, respectively], using a median split of EFT scores.)
Of broader contextual interest, we note that there has been a relatively large literature in which an attempt has been made to establish a link between gender and performance on spatial tasks. In fact, such a link is often assumed; for example, Shaffer (2000) states that "boys outperform girls on tests of visual/spatial abilities," and in Sherman (1967) we find the assertion that "key measures of analytical cognitive approach are substantially related to space perception, and therefore are sex biased." However, although such differences are sometimes reported, their stability is questionable: Although in some previous results females' completion times on the EFT were longer than those of male subjects (e.g., Annett, 1992; Freedman \& Rovegno, 1981; Geiringer \& Hyde, 1976; Goldstein \& Chance, 1965; Linn \& Peterson, 1985; Sherman, 1967; Witkin, 1949b; Witkin et al., 1954), there are others in which a gender difference has failed to occur (Brosnan, 1998; Chance \& Goldstein, 1971; Crandall \& Sinkeldam, 1964; Goldstein \& Chance, 1965; Hall, Gregory, Billinger, \& Fisher, 1988; Harley, Kalish, \& Silverman, 1974; O’Donnell, Paulsen, \& McGann, 1978; Petrusic, Varro, \& Jamieson, 1978; Weiner, 1955; Williams, LaPointe, \& Riski, 1974). Some examples specific to the EFT are the following: Crandall and Sinkeldam (1964) found no significant difference between girls and boys in a study of children's socially and perceptually dependent behaviors, including EFT performance; Hall et al. (1988) found age, but not gender, differences in the EFT; and Harley et al. (1974) found gender differences in the RFT, but not in the EFT. Of particular significance, Goldstein and Chance (1965) and Chance and Goldstein (1971) found that initially present gender differences in EFT performance disappeared if subjects were allowed some familiarity with the task, and they suggested that previously found gender differences on the EFT might have been due to the learning history of the experimental subjects.

7. The discordances for VPV and VPEL do differ in several ways. (1) The visual stimulation by the inducing stimulus that is discordant with the gravitational stimulation deviates along a different physical dimension: visual pitch for VPEL and frontal plane orientation for the setting of a rod to VPV. (2) The discrimination that is subject to induction itself lies along a different perceptual dimension in the two cases: elevation for the VPEL setting and frontal plane orientation for the VPV setting. (3) For VPV, the dimensions of variation for the inducing stimulus and the test stimulus are identical, whereas for the VPEL setting they are different.

8. Several other measures related to egocentric space perception were shown not to correlate with EFT in the earliest reports by Witkin et al. (1954).

9. The three sets of correlations between VPV and VPEL were as follows. In Poquin et al. (1998), $r=+.67$, in an experiment in which VPEL was measured by the subject's setting of the elevation of a very short horizontal bar in the presence of a large, variably pitched square inducing frame (five pitches in the range of $\pm 15^{\circ}$ ) in otherwise total darkness, VPV was measured by setting a long rod to appear vertical in the median plane when the same frame was variably roll tilted (five roll tilts in the range of $\pm 15^{\circ}$ ); the +.67 correlation was between the slopes of the VPEL-versus-pitch and VPV-versus-roll-tilt functions for the 14 subjects. In two separate experiments, employing a large square frame (first experiment, binocular, 49 subjects; second experiment, monocular, 57 subjects), Guzy et al. (2003) reported that the correlations between VPV and VPEL were $r=+.38$ and $r=+.29$, respectively; their procedure differed from Poquin et al.'s in that, for three pitch orientations of the frame $\left( \pm 22^{\circ}\right.$ and $\left.0^{\circ}\right)$, they employed the same long line (horizontally oriented) to measure VPEL as that employed for measuring VPV (three roll tilt orientations, $\pm 22^{\circ}$ and $0^{\circ}$ ). The correlation between VPV and VPEL (monocular viewing) in Shavit et al.'s (2004) report was +.45 ; see notes 10 and 11 .

10. Some background will be useful here. Previous work (Matin \& Li, 1992a, 1994c) had described spatial induction of large changes in both VPV and VPEL that resulted from stimulation from the same eccentrically located individual lines in otherwise total darkness (one-line stimuli) or from pairs of these single, bilaterally symmetrically located lines (two-line stimuli). However, the rules of combination of one-line stimuli into two-line stimuli differ for VPV and VPEL: Two lines located bilaterally symmetrically with the same orientation have identical influences on VPV but opposite influences on VPEL, and consequently, simultaneous presentation of two such lines produces a length-dependent increase in the effect on VPV over that of the single line but results in no influence 
on VPEL; two lines located bilaterally symmetrically with equal and opposite orientations have opposite influences on VPV but identical influences on VPEL; consequently, simultaneous presentation of two such lines results in no influence on VPV but produces a length-dependent increase in the influence on VPEL. Furthermore, it is not necessary that the individual inducing lines arise from pitched planes for VPEL to be influenced or that they arise from the frontoparallel plane for VPV to be influenced, as was done in the work by Poquin et al. (1998) and Guzy et al. (2003) with the square frames. For any line in a pitched plane, there is a corresponding unique line in a frontoparallel plane that stimulates the same retinal orientation, and for the monocularly viewing subject, such corresponding lines from the two planes generate indistinguishable influences on VPEL, as well as indistinguishable influences on VPV; it was thus possible to measure the influences of the very same set of variably oriented individual lines from the frontoparallel plane on both VPEL and VPV as one-line stimuli and also present bilaterally symmetrically located pairs of these same one-line stimuli simultaneously as variably oriented two-line stimuli (see also Li \& Matin, 2005a, 2005b, for comparisons between the influences of the full four-sided square frame and the one-line and two-line stimuli, as well as several other configurations). A significant bottom line here is that the entire square frame adds very little to the induction on VPV beyond that generated by the one-line and two-line stimuli.

11. Measurements were made on an additional 10 subjects subsequent to the 2004 report; the two-line VPV/VPEL slope-versus-slope correlation for all 30 subjects was +.57 ; for the right and left one-line conditions, the values were +.57 and -.44 , respectively.

(Manuscript received July 22, 2004; revision accepted for publication May 26, 2005.) 